\title{
Daten und Methoden des Deutschen Freiwilligensurveys
}

\author{
Julia Simonson, Nora Karnick, Nadiya Kelle \& Nicole Hameister
}

\section{Kernaussagen}

Der Deutsche Freiwilligensurvey (FWS) ist eine repräsentative telefonische Befragung zum freiwilligen Engagement in Deutschland. Der Freiwilligensurvey wurde bislang fünfmal durchgeführt (1999: $\mathrm{n}=14.922$, 2004: $\mathrm{n}=15.000,2009: \mathrm{n}=20.005$, 2014: $n=28.690$ und 2019: $n=27.762$ ). Grundgesamtheit ist die Wohnbevölkerung Deutschlands in Privathaushalten im Alter ab 14 Jahren.

Die Telefoninterviews für den Freiwilligensurvey 2019 wurden wie bereits 2014 über Festnetz und über Mobilfunk geführt. Insgesamt wurden 19.498 Interviews über das Festnetz (70,2 Prozent) und 8.264 Interviews über Mobiltelefone geführt (29,8 Prozent).

Neben Interviews in Deutsch waren im Freiwilligensurvey 2019 wie bereits 2014 auch Interviews in weiteren Sprachen möglich. Die Befragten hatten neben Deutsch die Auswahl zwischen Englisch, Arabisch, Russisch, Türkisch und Polnisch. Insgesamt wurden 608 nicht deutschsprachige Interviews geführt.

Alle Angaben zum freiwilligen Engagement wurden 2019 wie 2014 anhand eines Kriterienkatalogs geprüft. Ziel dieser Prüfung war es, diejenigen Tätigkeiten zu identifizieren, die den Kriterien für freiwilliges Engagement eindeutig widersprechen. Im Falle eines solchen Widerspruchs wurde eine Tätigkeit nicht als freiwilliges Engagement gewertet und entsprechend recodiert. Im Freiwilligensurvey 2019 wurden 2,6 Prozent der Personen, die sich selbst zunächst als engagiert eingeschätzt haben, als nicht-engagiert gewertet. 
Das Gewichtungskonzept wurde für den Freiwilligensurvey 2019 angepasst, indem zusätzlich die Bildungsinformation einbezogen wird. Die Gewichtung inklusive Bildung wird durchgängig für die Analysen des Berichts verwendet, im Zeitvergleich auch rückwirkend für die bisherigen Erhebungswellen. Dadurch ergeben sich für alle Erhebungswellen niedrigere Engagementquoten als bisher berichtet, und zwar um jeweils drei bis vier Prozentpunkte. Es ändert sich somit insgesamt das Niveau der Engagementquoten, der Trend bleibt jedoch im Wesentlichen bestehen. 


\subsection{Einleitung}

Der Deutsche Freiwilligensurvey (FWS) ist eine repräsentative telefonische Befragung zum freiwilligen Engagement in Deutschland, die bislang fünfmal durchgeführt wurde (1999: $n=14.922,2004: n=15.000,2009: n=20.005,2014: n=28.690$ und 2019: $n=27.762$ ). Der Deutsche Freiwilligensurvey wird vom Bundesministerium für Familie, Senioren, Frauen und Jugend (BMFSFJ) gefördert. Die wissenschaftliche Leitung des Freiwilligensurveys 2019 liegt wie schon bei der vierten Erhebungswelle beim Deutschen Zentrum für Altersfragen (DZA). Die Datenerhebung des Freiwilligensurveys 2019 wurde - wie bereits die Erhebung 2014 - von infas Institut für angewandte Sozialwissenschaft durchgeführt. Die Datenerhebungen der Jahre 1999, 2004 und 2009 wurden von TNS Infratest durchgeführt.

Grundgesamtheit des Freiwilligensurveys ist die Wohnbevölkerung Deutschlands in Privathaushalten im Alter ab 14 Jahren. Die Telefoninterviews werden über Festnetz und (seit der vierten Erhebungswelle) auch über Mobilfunk geführt und finden auf Deutsch sowie (ebenfalls seit der vierten Welle) in fünf weiteren Sprachen statt (Englisch, Arabisch, Russisch, Türkisch, Polnisch). Im Jahr 2019 wurden 27.762 Personen befragt. Dabei wurde die Basisstichprobe mit einer Fallzahl von n = 24.058 um eine durch die Bundesländer finanzierte länderspezifische Aufstockung von n = 3.704 ergänzt.

In diesem Kapitel werden die methodischen Grundlagen des Freiwilligensurveys 2019 dargestellt. Es wird das Vorgehen bei der Datenerhebung dargelegt und es wird erläutert, wie bei der Datenaufbereitung und bei der Prüfung der offenen Angaben zum freiwilligen Engagement vorgegangen wurde. Die Stichprobe und das Vorgehen bei der Gewichtung werden beschrieben und die Analysestrategie für den Bericht mit den zentralen Differenzierungskriterien dargestellt. Abschließend werden die Möglichkeiten der Datennutzung über das Forschungsdatenzentrum des DZA (FDZDZA) vorgestellt.

\subsection{Vorbereitende Arbeiten für den Freiwilligensurvey 2019}

Das Erhebungsinstrument für den Freiwilligensurvey 2019 wurde in einem partizipativen Prozess weiterentwickelt. Zu Beginn dieses Prozesses wurden Akteure des zivilgesellschaftlichen Engagements und der Zivilgesellschaftsforschung vom BMFSFJ eingeladen an der Weiterentwicklung des Freiwilligensurveys 2019 mitzuwirken. Zu diesem Zweck wurde im Juni 2017 ein Workshop veranstaltet, auf dem Vorschläge eingebracht und diskutiert werden konnten. Diese Vorschläge wurden durch Anregungen im Rahmen eines Workshops mit Datennutzenden am DZA im November 2017 und von verschiedenen Gremien wie beispielsweise dem Forum Zivilgesellschaftsdaten sowie dem Beirat des Freiwilligensurveys 2019 ergänzt. Da für die Interviewdauer 2019, wie auch 2014, durchschnittlich 30 Minuten geplant waren, 
konnte nur ein Teil aller Anregungen umgesetzt werden. Für Neuaufnahmen wurden zudem Fragen, die im Freiwilligensurvey 2014 gestellt worden waren, aus dem Erhebungsinstrument gestrichen. Dabei galt das Prinzip der Wahrung von Kontinuität bei gleichzeitiger Fortentwicklung des Fragebogens. Der Prozess der Weiterentwicklung des Freiwilligensurveys hat unter anderem zur Neuaufnahme folgender Themen geführt: Einstellungen zur Demokratie und Institutionenvertrauen, Engagement für Geflüchtete und von Geflüchteten, Formen der Nutzung des Internets für das Engagement und Spendenzwecke (siehe Kapitel 1).

In Vorbereitung auf die Haupterhebung wurden zwei Pretests durchgeführt. Im Zeitraum von Oktober bis November 2018 fand der Pretest für das deutschsprachige Erhebungsinstrument statt. Ein zentrales Ergebnis des deutschsprachigen Pretests war, dass das Instrument noch zu umfangreich war und gekürzt werden musste, um die anvisierte Befragungszeit von durchschnittlich 30 Minuten zu realisieren. Die Pretests in den fünf weiteren Sprachen zielten darauf ab, die Praktikabilität der übersetzten Instrumente zu testen und fanden im Zeitraum von Februar bis März 2019 statt. Die Haupterhebung wurde vom 18. März 2019 bis zum 16. November 2019 durchgeführt.

\subsection{Ablauf des Interviews und Befragungsinhalte}

Die computerunterstützten Telefoninterviews (CATI) fanden sowohl über das Festnetz als auch über Mobilfunk statt. Unabhängig davon, ob es sich um ein Festnetzoder Mobilfunkinterview handelte, verlief das Interview identisch; lediglich bei der Auswahl der Befragungsperson wurde unterschiedlich vorgegangen. Bei den Festnetzinterviews wurde die Zielperson aus dem Haushalt nach dem Last-BirthdayVerfahren ausgewählt, also diejenige Person ab 14 Jahren im Haushalt, die zuletzt Geburtstag hatte. Bei den Mobilfunkinterviews war hingegen kein weiterer Auswahlschritt erforderlich, da es sich in der Regel um individuell vergebene Rufnummern und damit um eine Personenstichprobe handelte. Hier wurde die Person befragt, die das Telefon hauptsächlich nutzt. Darüber hinaus wurde bei den Mobilfunkinterviews nach dem Bundesland des Hauptwohnsitzes gefragt. In den Festnetzinterviews war diese Abfrage nicht notwendig, da die Länderkennung bereits Teil der Stichprobeninformation war (Schiel et al. 2020).

Das Interview startete mit allgemeinen Fragen zur Person. Zunächst wurde das Geschlecht der befragten Person durch die Interviewperson eingeschätzt; dies entspricht dem bisherigen Vorgehen im Freiwilligensurvey. Erstmals konnte in der Befragung 2019 auch die Geschlechterangabe ,divers' gemacht werden (detaillierte Informationen zur Erhebung des Geschlechts in Abschnitt 2.10). Weitere Fragen zu Beginn des Interviews richteten sich auf soziodemografische Merkmale wie Alter, Migrationserfahrung, Haushaltszusammensetzung, Erwerbsstatus und Bildungshintergrund; zudem wurden Fragen zur Vereinsmitgliedschaft und zur politischen Partizipation gestellt. 
Kasten 2-1 Frage zur Aktivität in vierzehn gesellschaftlichen Bereichen

Es gibt vielfältige Möglichkeiten, außerhalb von Beruf und Familie irgendwo mitzumachen, beispielsweise in einem Verein, einer Initiative, einem Projekt oder einer Selbsthilfegruppe. Ich nenne Ihnen verschiedene Bereiche, die dafür in Frage kommen. Wenn Sie an die letzten 12 Monate denken: Haben Sie sich in einem oder mehreren dieser Bereiche aktiv beteiligt? Sind Sie oder waren Sie irgendwo aktiv...

1) ... im Bereich Sport und Bewegung z. B. in einem Sportverein oder in einer Bewegungsgruppe?

2) ... im Bereich Kultur und Musik z. B. einer Theater- oder Musikgruppe, einem Chor, einer kulturellen Vereinigung oder einem Förderverein?

3) ... im Bereich Freizeit und Geselligkeit z. B. in einem Kleingartenverein oder einem Spieletreff?

4) ... im sozialen Bereich z. B. in einem Wohlfahrtsverband oder einer anderen Hilfsorganisation, in der Nachbarschaftshilfe oder einer Selbsthilfegruppe?

5) ... im Gesundheitsbereich z. B. als Helfer/in der Krankenpflege oder bei Besuchsdiensten, in einem Verband oder einer Selbsthilfegruppe?

6) ... im Bereich Schule oder Kindergarten z. B. in der Elternvertretung, der Schülervertretung oder einem Förderverein?

7) ... in der außerschulischen Jugendarbeit oder der Bildungsarbeit für Erwachsene z. B. Kinderoder Jugendgruppen betreuen oder Bildungsveranstaltungen durchführen?

8) ... im Bereich Umwelt, Naturschutz oder Tierschutz z. B. in einem entsprechenden Verband oder Projekt?

9) ... im Bereich Politik und politische Interessenvertretung z. B. in einer Partei, im Gemeinderat oder Stadtrat, in politischen Initiativen oder Solidaritätsprojekten?

10) ... im Bereich der beruflichen Interessenvertretung außerhalb des Betriebes $z$. B. in einer Gewerkschaft, einem Berufsverband, einer Arbeitsloseninitiative?

11) ... im kirchlichen oder religiösen Bereich z. B. in der Kirchengemeinde, einer religiösen Organisation oder einer religiösen Gemeinschaft?

12) ... im Bereich der Justiz und der Kriminalitätsprobleme z. B. als Schöffe/in oder Ehrenrichter/in, in der Betreuung von Straffälligen oder Verbrechensopfern?

13) ... im Unfall- oder Rettungsdienst oder in der freiwilligen Feuerwehr?

14) ... in einem bisher noch nicht genannten Bereich z. B. in Bürgerinitiativen oder Arbeitskreisen zur Orts- und Verkehrsentwicklung, aber auch Bürgerclubs und sonstiges, das bisher nicht genannt wurde?

Die Abfrage zum freiwilligen Engagement erfolgte wie in den vorangegangenen Erhebungswellen zweistufig. Im ersten Schritt wurden Aktivitäten außerhalb von Beruf und Familie erfragt. Für die Ermittlung dieser gemeinschaftlich öffentlichen Aktivitäten wurden in der Befragung vierzehn Gesellschaftsbereiche benannt, in denen man "mitmachen“ oder sich „aktiv beteiligen“ kann (siehe Kasten 2-1).

Die Bereiche wurden auf die gleiche Weise auch in den vorangegangenen Erhebungen seit 2004 erfasst, allerdings wurden teilweise Veränderungen an den Bereichsbeispielen vorgenommen. Im Freiwilligensurvey 1999 wurde neben den aufgeführten Bereichen zusätzlich der Bereich der wirtschaftlichen Selbsthilfe abgefragt. Dieser wurde jedoch nur von sehr wenigen Befragten angegeben und daher in den folgenden Erhebungen nicht mehr berücksichtigt.

Für jeden der Bereiche, für die eine Person angegeben hatte aktiv zu sein, wurde nachfolgend abgefragt, ob dort in den letzten zwölf Monaten freiwillig und un- 
Kasten 2-2 Frage zum freiwilligen Engagement in vierzehn gesellschaftlichen Bereichen

Uns interessiert nun, ob Sie in den Bereichen, in denen Sie aktiv sind, auch ehrenamtliche Tätigkeiten ausüben oder in Vereinen, Initiativen, Projekten oder Selbsthilfegruppen engagiert sind. Es geht um freiwillig übernommene Aufgaben und Arbeiten, die man unbezahlt oder gegen geringe Aufwandsentschädigung ausübt. Sie sagten, Sie sind im Bereich [Name des Bereichs] aktiv. Wenn Sie an die letzten 12 Monate denken:

Haben Sie in diesem Bereich auch Aufgaben oder Arbeiten übernommen, die Sie freiwillig oder ehrenamtlich ausüben?

Kasten 2-3 Offene Fragen zu den freiwilligen Tätigkeiten

1) Um welche Art von Gruppe, Organisation oder Einrichtung handelt es sich, in der Sie da tätig sind? Können Sie mir das bitte kurz beschreiben?

2) Und was machen Sie dort konkret? Welche Aufgabe oder Arbeit üben Sie dort aus?

3) Hat Ihre Tätigkeit einen Namen? Wenn ja, welchen?

entgeltlich oder gegen eine geringe Aufwandsentschädigung Aufgaben oder Arbeiten übernommen wurden (siehe Kasten 2-2). Wurden freiwillige oder ehrenamtliche Aufgaben oder Arbeiten von der befragten Person übernommen, galt sie für den weiteren Verlauf des Interviews als freiwillig engagiert. Die Frage nach übernommenen Aufgaben oder Arbeiten ist im Kasten 2-2 dargestellt.

Wurde auch diese Frage bejaht, folgten drei weitere Fragen zum Inhalt jeder einzelnen freiwilligen Tätigkeit mit offenen Antwortmöglichkeiten (die Interviewenden notierten die Antworten in Sätzen oder Stichworten; siehe Kasten 2-3).

Für jeden Bereich, in dem eine Person angegeben hatte, aktiv zu sein, konnten bis zu drei freiwillige Tätigkeiten angegeben werden. Anschließend wurde bereichsübergreifend nachgefragt, ob noch weitere nicht genannte freiwillige Tätigkeiten übernommen wurden. Hierbei konnten bis zu drei weitere Tätigkeiten genannt werden. Insgesamt konnten somit bis zu 45 unterschiedliche freiwillige Tätigkeiten angegeben werden. Mit 48,9 Prozent gab knapp die Hälfte der Engagierten an, genau eine freiwillige Tätigkeit auszuüben. Jeweils etwa ein Viertel der Engagierten gab zwei freiwillige Tätigkeiten (26,6 Prozent) beziehungsweise drei oder mehr freiwillige Tätigkeiten an (24,5 Prozent). Nachdem alle freiwilligen Tätigkeiten erfasst wurden, folgte eine Prüfung durch die Befragten selbst. Es wurde nachgefragt, ob die angegebenen Tätigkeiten tatsächlich freiwillig oder ehrenamtlich ausgeübt werden und - bei mehreren angegebenen Tätigkeiten - ob es sich um unterschiedliche Aufgaben handelt.

Wie bereits im Freiwilligensurvey 2014 wurden die Fragen zu Aktivitäten und Engagement unter Verwendung eines Zeitfensters („in den letzten zwölf Monaten“) gestellt, um saisonale Schwankungen auszugleichen. Die Angaben beziehen sich also 
auf die jeweils letzten zwölf Monate vor der Befragungsteilnahme. In den ersten drei Erhebungswellen des Freiwilligensurvey gab es bei diesen Fragen keinen vorgegebenen zeitlichen Bezugsrahmen, es wurde nach Aktivitäten und Engagement „derzeit“ gefragt. Vergleichsuntersuchungen (Kelle et al. 2021a) deuten darauf hin, dass die Engagementquote im Freiwilligensurvey bei der Abfrage unter Verwendung des Zeitfensters von zwölf Monaten etwas höher ausfällt als bei der Abfrage ohne festen Zeitrahmen. Möglicherweise werden unregelmäßig ausgeübte Tätigkeiten bei der Abfrage mit diesem klar definierten Zeitfenster besser erfasst als bei der Abfrage ohne klare Zeitangabe. Vermutlich denken Befragte bei einer zeitlich unspezifischen Abfrage eher an ein kleineres Zeitfenster und berichten somit weiter zurückliegende beziehungsweise unregelmäßig ausgeübte Tätigkeiten anteilig seltener (siehe Kapitel 8). Der deutliche Anstieg der Engagementquote zwischen den Erhebungsjahren 2009 und 2014 ist daher vermutlich teilweise auch auf diese methodische Veränderung zurückzuführen (siehe hierzu auch Kapitel 3).

Nach der zweistufigen Abfrage zum freiwilligen Engagement hing der weitere Verlauf des Interviews davon $\mathrm{ab}$, ob die befragte Person angab freiwillig engagiert zu sein oder nicht (Abbildung 2-1). Die Folgefragen bezogen sich entweder auf die freiwillige(n)Tätigkeit(en) (bei Engagierten) oder auf früheres Engagement, auf Beendigungs- beziehungsweise Hinderungsgründe für freiwilliges Engagement sowie auf die Bereitschaft, sich zukünftig zu engagieren (bei Nicht-Engagierten). Zudem gab es

Abbildung 2-1 Ablauf des Interviews

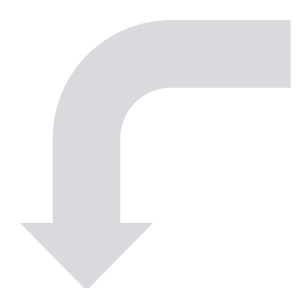

Engagierte Befragte

zum Beispiel

Engagement insgesamt

Zeitaufwendigste freiwillige Tätigkeit

Kontext des Engagement

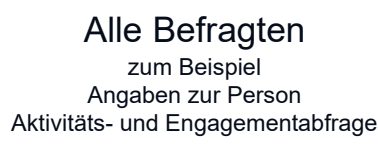

Aktivitäts- und Engagementabfrage

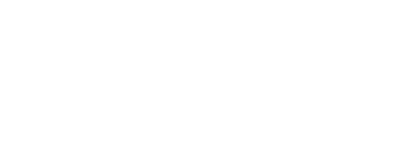

Nicht-engagierte Befragte

zum Beispiel

Früheres Engagement

Engagementbereitschaft

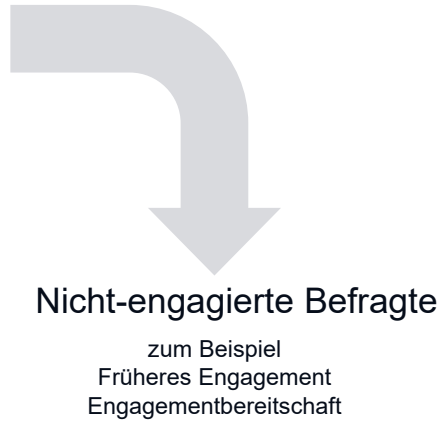

Quelle: FWS 2019. Eigene Darstellung (DZA).

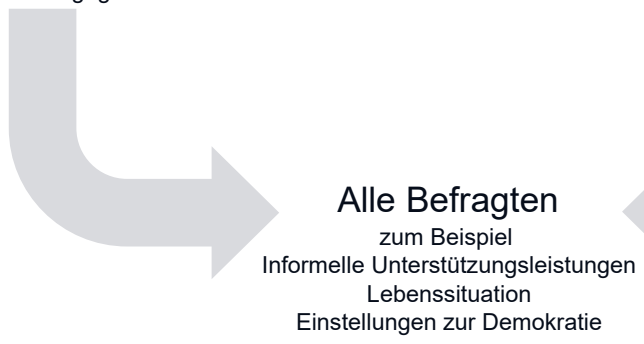


einen Frageblock, der Engagierten spezifisch zu ihrer einzigen oder - bei mehreren Tätigkeiten - zeitaufwendigsten Tätigkeit gestellt wurde. Fragen zu informellen Unterstützungsleistungen, zur Gesundheit, zur finanziellen Situation, zu Geldspenden sowie zu Einstellungen gegenüber der Demokratie und zum Institutionenvertrauen wurden dann wieder allen Befragten gestellt.

\subsection{Datenaufbereitung und Prüfung der offenen Angaben zum freiwilligen Engagement}

Datenprüfung und -aufbereitung sind Arbeitsschritte, die notwendig sind, um einen Arbeitsdatensatz für die Analysen zu erhalten, auf denen die im vorliegenden Bericht dargestellten Ergebnisse basieren. So wurden beispielsweise die Variablen für die verwendeten Differenzierungsmerkmale aufbereitet. Dafür wurden für die Erstellung der Altersgruppen die kategoriale und die offene Abfrage und für die Erstellung der Bildungsgruppen sowie die differenzierte Einteilung nach Migrationshintergrund jeweils die Angaben aus mehreren Interviewfragen zusammengefasst (siehe Abschnitt 2.10). Einen zentralen Stellenwert in der Datenaufbereitung hatte die umfangreiche Prüfung der offenen Angaben, die die Befragten zur Organisation, zu Aufgaben und zum Namen ihrer freiwilligen Tätigkeiten machten (siehe Kasten 2-3 in Abschnitt 2.3). Diese offenen Angaben der Personen zu ihrem Engagement wurden während des Interviews durch die Befragten selbst und während der Datenaufbereitung anhand eines Kriterienkatalogs (Tabelle 2-1) daraufhin geprüft, ob die angegebene Tätigkeit tatsächlich als freiwilliges Engagement gezählt werden kann.

Während der Prüfung im Zuge der Datenaufbereitung wurde für alle Kriterien eine Negativprüfung vorgenommen, das heißt, es wurde geprüft, ob die offenen Angaben zur freiwilligen Tätigkeit den Kriterien für Engagement widersprechen. Bei der Erstellung des Kriterienkatalogs wurde sich an den fünf Bestimmungsstücken der Enquete-Kommission „Zukunft des bürgerschaftlichen Engagements" orientiert (Deutscher Bundestag 2002). Lagen keine eindeutigen Hinweise auf eine Verletzung der Kriterien vor oder fehlten Angaben dazu, wurde die Tätigkeit als Engagement gewertet. Wurde hingegen mindestens eines der Kriterien verletzt, wurde die Tätigkeit nicht als Engagement gewertet. Wenn Zweifel vorlagen, ob es sich bei einer Tätigkeit um Engagement handelt oder nicht, zählte die Selbsteinschätzung der interviewten Person, und die Tätigkeit wurde als Engagement gewertet.

Das Kriterium der Freiwilligkeit wurde bereits während des Interviews im Rahmen einer standardisierten Nachfrage geprüft. Darüber hinaus gab es zum Zeitpunkt der Befragung keine verpflichtenden Dienste in Deutschland, sodass auf eine systematische Prüfung der Freiwilligkeit anhand der offenen Angaben verzichtet werden konnte.

Eine Einschätzung des Nutzens der freiwilligen Tätigkeit für das Gemeinwohl war nicht möglich, da diese Information nicht im Rahmen der Abfrage der freiwilligen Tätigkeit erfasst wurde. Dies hängt insbesondere damit zusammen, dass es sich bei der 
Tabelle 2-1 Kriterienkatalog für die Überprüfung der offenen Angaben

\begin{tabular}{|c|c|c|c|}
\hline Kriterium & $\begin{array}{l}\text { Tätigkeit wird als Enga- } \\
\text { gement gewertet, wenn } \\
\text { keine der Aussagen ver- } \\
\text { letzt ist. }\end{array}$ & $\begin{array}{l}\text { Tätigkeit wird nicht als } \\
\text { Engagement gewertet, } \\
\text { wenn mindestens eine } \\
\text { der Aussagen erfüllt ist. }\end{array}$ & $\begin{array}{l}\text { Beispiele für nicht als } \\
\text { Engagement gewertete } \\
\text { Tätigkeiten }\end{array}$ \\
\hline Aktiv & $\begin{array}{l}\text { Es handelt sich bei der } \\
\text { Angabe um eine Tätigkeit. }\end{array}$ & $\begin{array}{l}\text { Es handelt sich bei der } \\
\text { Angabe um eine passive } \\
\text { Mitgliedschaft oder um } \\
\text { eine Spendentätigkeit. }\end{array}$ & $\begin{array}{l}\text { Mitglied im Förderverein; } \\
\text { Blutspender }\end{array}$ \\
\hline $\begin{array}{l}\text { Unentgelt- } \\
\text { lich }\end{array}$ & $\begin{array}{l}\text { Die Tätigkeit ist nicht } \\
\text { auf materiellen Gewinn } \\
\text { gerichtet. }\end{array}$ & $\begin{array}{l}\text { Es handelt sich um eine } \\
\text { berufliche, bezahlte oder } \\
\text { innerbetriebliche Tätigkeit } \\
\text { innerhalb der Arbeitszeit. }\end{array}$ & $\begin{array}{l}\text { Praktikum in Autowerkstatt; } \\
\text { Betriebsrat; Ausbildung zur } \\
\text { Altenpflegerin }\end{array}$ \\
\hline Öffentlich & $\begin{array}{l}\text { Die Tätigkeit findet im } \\
\text { öffentlichen Raum statt. }\end{array}$ & $\begin{array}{l}\text { Es handelt sich um eine } \\
\text { Tätigkeit in der Familie } \\
\text { oder im Freundeskreis. }\end{array}$ & $\begin{array}{l}\text { Pflege der Mutter; Kümmere } \\
\text { mich um ein Kind einer } \\
\text { Freundin }\end{array}$ \\
\hline $\begin{array}{l}\text { Gemein- } \\
\text { schaftlich }\end{array}$ & $\begin{array}{l}\text { Die Tätigkeit wird gemein- } \\
\text { schaftlich beziehungswei- } \\
\text { se kooperativ ausgeübt. }\end{array}$ & $\begin{array}{l}\text { Es handelt sich um die } \\
\text { Ausübung eines Hobbys } \\
\text { ohne kooperativen Team- } \\
\text { charakter. }\end{array}$ & $\begin{array}{l}\text { Kleingarten - halte meinen } \\
\text { Garten in Schuss; Volkshoch- } \\
\text { schule - bilde mich weiter }\end{array}$ \\
\hline
\end{tabular}

Quelle: FWS 2019. Eigene Darstellung (DZA).

Gemeinwohlorientierung um ein schwer zu operationalisierendes Konzept handelt: Der Begriff der Gemeinwohlorientierung kann vor dem Hintergrund unterschiedlicher Norm- und Wertvorstellungen von Personen sehr unterschiedlich, ja konträr verstanden werden. Eine Tätigkeit, die vor dem Hintergrund eines bestimmten Wertesystems als, gemeinwohlorientiert' verstand wird, kann vor dem Hintergrund eines konkurrierenden Wertesystems als ,gemeinwohlschädigend' interpretiert werden. Darüber hinaus ist davon auszugehen, dass eine Abfrage der Gemeinwohlorientierung starken Effekten sozialer Erwünschtheit unterliegen würde. Die Gemeinwohlorientierung des Engagements wurde daher weder in der Erhebungswelle 2019, noch in den davorliegenden Erhebungsjahren geprüft; sie wurde vielmehr seit der ersten Welle des Freiwilligensurveys als gegeben vorausgesetzt.

Bevor die Prüfung der offenen Angaben zu den freiwilligen Tätigkeiten durch das DZA vorgenommen wurde, kam es bereits während des Interviews zu einer Prüfung durch die Befragten selbst. Die befragten Personen, die Angaben zu freiwilligen Tätigkeiten machten, wurden anschließend gefragt, ob die angegebenen Tätigkeiten tatsächlich freiwillig und unentgeltlich (oder gegen eine geringe Aufwandsentschädigung) ausgeübt wurden. Hierzu wurde 2014 und 2019 eine standardisierte Frage gestellt. In den vorangegangenen Erhebungswellen gab es diese standardisierte Abfrage nicht; hier entschieden zum Teil die interviewenden Personen und nicht die Befragten selbst darüber, ob eine Tätigkeit als freiwilliges Engagement einging oder 
Tabelle 2-2 Anteil der nach Prüfung als,nicht-engagiert' gewerteten Personen an allen Personen, die sich selbst zunächst als engagiert einschätzten, im Zeitvergleich 1999-2019, in Prozent

\begin{tabular}{lccccc}
\hline & FWS 1999 & FWS 2004 & FWS 2009 & FWS 2014 & FWS 2019 \\
\hline $\begin{array}{l}\text { Prüfung während des Interviews: } \\
\begin{array}{l}\text { Anteil der als,nicht-engagiert' } \\
\text { eingeschätzten Personen }\end{array}\end{array}$ & $--^{-a}$ & $--^{-a}$ & 8,2 & 0,8 & 1,4 \\
$\begin{array}{l}\text { Prüfung nach dem Interview: An- } \\
\text { teil der als, nicht-engagiert' ein- }\end{array}$ & $--^{-a}$ & $--^{-a}$ & 1,9 & 0,8 & 1,3 \\
geschätzten Personen & & & & & \\
\hline $\begin{array}{l}\text { Prüfung insgesamt: Gesamtpro- } \\
\text { zent der als, nicht-engagiert' ein- } \\
\text { geschätzten Personen }\end{array}$ & $3,5^{\text {b }}$ & 5,4 & 10,1 & 1,6 & 2,6 \\
\hline
\end{tabular}

Quelle: FWS 1999, FWS 2004, FWS 2009, FWS 2014, FWS 2019. Basis: alle Engagierten nach Selbsteinschätzung vor Prüfung. Eigene Berechnung (DZA).

Hinweis: Es handelt sich bei den Prozentangaben um ungewichtete Anteile. Abweichungen in den Prozentwerten ergeben sich durch das Auf- und Abrunden der Werte.

${ }^{a}$ Für die Jahre 1999 und 2004 liegen keine differenzierten Informationen über die Zahl der Personen vor, die während des Interviews beziehungsweise nach dem Interview als, nicht- engagiert' eingeschätzt wurden.

${ }^{b}$ Die Angabe Gesamtprozent für 1999 wurde geschätzt; da für dieses Jahr lediglich Informationen über den Anteil der Recodierungen auf Tätigkeitsebene, nicht aber auf Personenebene vorliegen. Bei der Schätzung wurde davon ausgegangen, dass der Anteil der Recodierungen auf Tätigkeitsebene dem auf Personenebene entspricht.

nicht. Der Anteil der Personen, die während des Interviews von, engagiert` zu ,nichtengagiert' wechselten, unterscheidet sich zwischen den Erhebungswellen deutlich (Tabelle 2-2). Während für die Erhebungswellen 1999 und 2004 dazu keine Zahlen vorliegen, ist der Anteil 2009 mit 8,2 Prozent deutlich höher als 2014 und 2019 mit o,8 Prozent sowie 1,4 Prozent.

Die Prüfung der offenen Angaben nach dem Interview, die 2014 und 2019 anhand der in Tabelle 2-1 beschriebenen Kriterien vorgenommen wurde, zeigt hingegen kaum Unterschiede im Anteil der dadurch von ,engagierten' zu ,nicht-engagierten geänderten Fälle zwischen den Wellen 2009, 2014 und 2019 (Tabelle 2-2). Auch hier liegen für 1999 und 2004 keine Informationen vor.

Für die Vergleichbarkeit der Engagementquoten zwischen den fünf Erhebungswellen ist der Anteil der insgesamt (also während des Interviews und danach) von ,engagiert' zu ,nicht-engagiert' recodierten Personen zentral. Je nach Anteil der recodierten Fälle verringert sich die Höhe der Engagementquote der jeweiligen Befragungswelle mehr oder weniger stark. Diese ungewichteten Anteile lagen im Jahr 1999 bei 3,5 Prozent ${ }^{1}$ und im Jahr 2004 bei 5,4 Prozent. Für 2009 ergibt sich insgesamt ein

1 Der Anteil der 1999 von, engagiert' zu, nicht-engagiert' recodierten Personen wurde geschätzt; da für dieses Jahr lediglich Informationen über den Anteil der Recodierungen auf Tätigkeitsebene, nicht aber auf Personenebene vorliegen. Bei der Schätzung wurde davon ausgegangen, dass der Anteil der Recodierungen auf Tätigkeitsebene (3,5 Prozent) dem auf Personenebene (3,5 Prozent) entspricht. 
deutlich höherer Anteil von 10,1 Prozent. Im Jahr 2014 lag der Anteil bei 1,6 Prozent; 2019 liegt der Anteil bei 2,6 Prozent. Somit sind die Unterschiede zwischen den Erhebungswellen hinsichtlich der Gesamtprozente der als ,nicht-engagiert' eingeschätzten Personen sehr deutlich; diese Unterschiede haben Auswirkungen auf die Höhe der geprüften Engagementquoten: Je mehr Fälle von ,engagiert' ${ }^{\prime} \mathrm{zu}$,nicht-engagiert ${ }^{\star}$ verändert werden, desto stärker weicht die geprüfte Engagementquote nach unten von der ungeprüften $\mathrm{ab}$.

\subsection{Stichprobenzusammensetzung}

Für die Datenerhebung kamen zwei Teilstichproben zum Einsatz. Die erste Stichprobe setzte sich aus bundesweit gestreuten Festnetz- und Mobilfunknummern zusammen (Basisstichprobe). Die Fälle der Mobilfunkstichprobe wurden proportional, die Fälle der Festnetzstichprobe disproportional nach Bundesländern gezogen (Schiel

Abbildung 2-2 Stichprobenzusammensetzung des Freiwilligensurveys 2019, nach Ländern

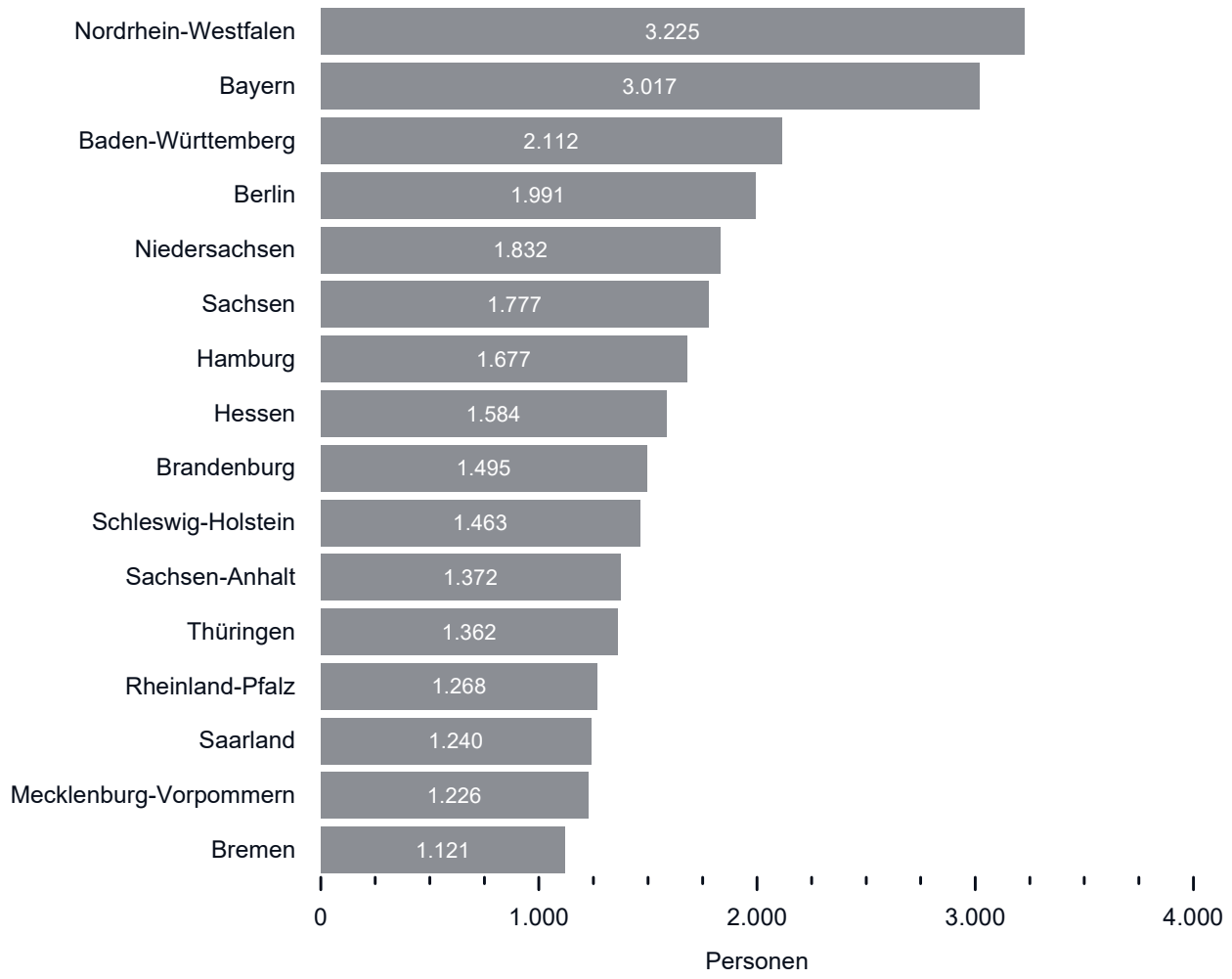


et al. 2020). Die angestrebte Verteilung in der Basisstichprobe betrug 30 Prozent Mobilfunkinterviews und 70 Prozent Festnetzinterviews, ${ }^{2}$ die realisierte Verteilung der Basisstichprobe 34,4 Prozent Mobilfunk- und 65,7 Prozent Festnetzinterviews. Darüber hinaus wurde eine disproportional nach Bundesländern geschichtete Zusatzstichprobe von Festnetznummern gezogen, um für Auswertungen auf Bundeslandebene auch für einwohnerschwächere Länder ausreichende Fallzahlen zu erreichen (Länderaufstockung). Im Rahmen der Basisstichprobe wurden 24.058 Interviews realisiert, im Rahmen der Länderaufstockung 3.704 Fälle. Bezogen auf die Gesamtstichprobe des Freiwilligensurveys 2019 (Basis und Länderaufstockung) wurden 19.498 Interviews über das Festnetz (70,2 Prozent) und 8.264 Interviews über Mobiltelefone geführt (29,8 Prozent).

Betrachtet man die Verteilung der Stichprobe auf die Länder (Abbildung 2-2), so wird ersichtlich, dass auch in den bevölkerungsschwächeren Bundesländern jeweils mehr als 1.000 Personen befragt werden konnten. Die hohen Stichprobenumfänge auf Länderebene ermöglichen es, für die jeweiligen Bundesländer vertiefende Analysen durchzuführen.

\subsection{Interviewsprachen}

Die Interviews des Freiwilligensurveys 2019 wurden auf Deutsch sowie in fünf weiteren Sprachen durchgeführt (Englisch, Arabisch, Russisch, Türkisch, Polnisch). Die Kontaktgespräche starteten zunächst auf Deutsch. Stellte sich heraus, dass die Kontaktperson nicht über ausreichende Deutschkenntnisse verfügt, wurde seitens der interviewenden Person versucht, die Sprache der befragten Person zu ermitteln. Handelte es sich um eine der vorgesehenen weiteren Interviewsprachen, wurde die befragte Person noch einmal durch eine Interviewperson mit entsprechender Muttersprache kontaktiert. Insgesamt wurden 638 Interviews geführt, bei denen der weitere Kontakt in einer der fünf weiteren Sprachen erfolgte. Bei 30 dieser Interviews wurde im Verlauf des Interviews allerdings wieder zur Interviewsprache Deutsch gewechselt. Somit wurden insgesamt 608 Interviews in nicht deutscher Sprache geführt (Tabelle 2-3).

Die im Freiwilligensurvey 2019 nach Deutsch mit Abstand am häufigsten genutzte Interviewsprache ist Englisch. Anzunehmen ist, dass diese Sprache nicht nur von Befragten mit Englisch als Muttersprache genutzt wurde, sondern auch von anderen Personen mit ausreichenden englischen Sprachkenntnissen. Die zweithäufigste nicht deutsche Interviewsprache ist Arabisch, gefolgt von Russisch, Türkisch und Polnisch.

2 Dieses Mischungsverhältnis von Festnetz- und Mobilfunknummern wird durch die Ergebnisse einer von infas durchgeführten Simulationsstudie nahegelegt (siehe Schiel et al. 2020). 
Tabelle 2-3 Fallzahlen und Anteile der nicht deutschsprachigen Interviews pro Sprache

\begin{tabular}{lcc}
\hline Interviewsprache & Anzahl & Anteil in Prozent \\
\hline Englisch & 229 & 37,7 \\
Arabisch & 154 & 25,3 \\
Russisch & 103 & 16,9 \\
Türkisch & 75 & 12,3 \\
Polnisch & 47 & 7,7 \\
\hline Gesamt nicht deutschsprachige Interviews & 608 & 100,0 \\
\hline Quelle: FWs 2019. Eigene Darstellung (DZA). Ungewichtete Fallzahlen und Anteile. & \\
Hinweis: Abweichungen in den Prozentwerten ergeben sich durch das Auf- und Abrunden der Werte.
\end{tabular}

\subsection{Stichprobenausschöpfung}

Die Interviews wurden als computerunterstützte Telefoninterviews (CATI) geführt. Tabelle 2-4 gibt die Fallzahlen zur Stichprobenausschöpfung an, einmal insgesamt (Spalte ,Gesamt') und einmal für die einzelnen Stichproben (Spalten ,Basis` und ,Aufstockung'). Die Bruttostichprobe im Freiwilligensurvey 2019 umfasste 1.557.784 eingesetzten Telefonnummern, die zufällig generiert wurden. Von diesen Anschlüssen gehörten 83,3 Prozent nicht zur Zielgruppe, so handelte es sich beispielsweise um Faxnummern oder der Anschluss existierte nicht. 5,3 Prozent bildeten eine unbekannte Auswahl, es konnten beispielsweise nur Anrufbeantworter erreicht werden oder der Anschluss war besetzt. Die weiteren 11,4 Prozent der Anschlüsse gehörten zur Zielgruppe ( $n=177.703$ ), also Privatpersonen der Wohnbevölkerung Deutschlands ab 14 Jahren.

Von den 177.703 Anschlüssen, die zur Zielgruppe gehörten, konnten wiederum nicht alle für ein Interview gewonnen werden. 0,3 Prozent davon waren nicht befragbar (zum Beispiel aus gesundheitlichen Gründen) und 15,9 Prozent konnten nicht erreicht werden; 83,9 Prozent der Zielgruppe konnten erreicht werden und waren befragbar $(n=149.053)$. Über Mobilfunk konnten prozentual deutlich mehr Personen nicht erreicht werden (21,6 Prozent) als über Festnetz (Basisstichprobe: 13,1 Prozent; Aufstockungsstichprobe: 15,4 Prozent).

Die Interviewenden hatten verschiedene Möglichkeiten, um Zielpersonen auch bei anfänglicher Ablehnung für die Befragung zu gewinnen. Dazu gehörten unter anderem eine Hilfeseite mit allen relevanten Informationen zur Studie sowie die Möglichkeit, den Personen Informationen zur Studie und eine Datenschutzerklärung per E-Mail zuzusenden. Es konnten zudem Wunschtermine für die Interviews vereinbart werden. 
Tabelle 2-4 Fallzahlen der Stichprobenausschöpfung im Freiwilligensurvey 2019, gesamt und nach Teilstichproben

\begin{tabular}{|c|c|c|c|c|c|}
\hline & \multirow[t]{2}{*}{ Gesamt } & \multicolumn{3}{|c|}{ Basis } & \multirow[t]{2}{*}{ Aufstockung } \\
\hline & & Gesamt & Festnetz & Mobilfunk & \\
\hline Bruttostichprobe (Fallzahlen) & 1.557 .784 & 1.340 .862 & 839.996 & 500.866 & 216.922 \\
\hline Bruttostichprobe (Prozentual) & 100,0 & 100,0 & 100,0 & 100,0 & 100,0 \\
\hline \multicolumn{6}{|l|}{ darunter: } \\
\hline nicht Zielgruppe & 83,3 & 83,3 & 82,4 & 84,8 & 83,0 \\
\hline unbekannte Auswahl & 5,3 & 5,3 & 5,5 & 4,9 & 5,5 \\
\hline bleibt: Zielgruppe & 11,4 & 11,4 & 12,0 & 10,3 & 11,5 \\
\hline Zielgruppe (Fallzahlen) & 177.703 & 152.828 & 101.134 & 51.694 & 24.875 \\
\hline Zielgruppe (Prozentual) & 100,0 & 100,0 & 100,0 & 100,0 & 100,0 \\
\hline \multicolumn{6}{|l|}{ darunter: } \\
\hline $\begin{array}{l}\text { Non-Response: nicht } \\
\text { befragbar }\end{array}$ & 0,3 & 0,2 & 0,4 & 0,0 & 0,4 \\
\hline $\begin{array}{l}\text { Non-Response: nicht } \\
\text { erreicht }\end{array}$ & 15,9 & 16,0 & 13,1 & 21,6 & 15,4 \\
\hline $\begin{array}{l}\text { bleibt: Zielgruppe erreicht } \\
\text { und befragbar }\end{array}$ & 83,9 & 83,8 & 86,6 & 78,4 & 84,3 \\
\hline $\begin{array}{l}\text { Zielgruppe erreicht und } \\
\text { befragbar (Fallzahlen) }\end{array}$ & 149.053 & 128.090 & 87.541 & 40.549 & 20.963 \\
\hline $\begin{array}{l}\text { Zielgruppe erreicht und } \\
\text { befragbar (Prozentual) }\end{array}$ & 100,0 & 100,0 & 100,0 & 100,0 & 100,0 \\
\hline \multicolumn{6}{|l|}{ darunter: } \\
\hline $\begin{array}{l}\text { Non-Response: } \\
\text { Verweigerung }\end{array}$ & 78,9 & 78,6 & 79,9 & 76,0 & 80,5 \\
\hline $\begin{array}{l}\text { Realisierte Interviews } \\
\text { darunter: }\end{array}$ & 21,1 & 21,4 & 20,1 & 24,0 & 19,5 \\
\hline $\begin{array}{l}\text { Interview vollständig reali- } \\
\text { siert und auswertbar }\end{array}$ & 18,6 & 18,8 & 18,0 & 20,4 & 17,7 \\
\hline $\begin{array}{l}\text { Zielgruppe erreicht und } \\
\text { befragbar, Interviews voll- } \\
\text { ständig realisiert und aus- } \\
\text { wertbar (Fallzahlen) }\end{array}$ & 27.762 & 24.058 & 15.794 & 8.264 & 3.704 \\
\hline
\end{tabular}

Quelle: FWS 2019; Methodenbericht zum FWS 2019 (Schiel et al. 2020), eigene Darstellung (DZA). 
Von den 149.053 Personen, die zur Zielgruppe gehörten, erreicht wurden und befragbar waren, haben 78,9 Prozent ihre Teilnahme verweigert (Tabelle 2-4). Über Mobilfunk haben prozentual etwas weniger Zielpersonen ihre Teilnahme verweigert (76,o Prozent) als über Festnetz (Basisstichprobe: 79,9 Prozent; Aufstockungsstichprobe: 8o,5 Prozent). Neben einer grundsätzlichen Verweigerung waren die Gründe beispielsweise, dass die Zielperson krank war, nur persönlich und nicht telefonisch befragt werden wollte oder keine Zeit hatte. Es konnten 21,1 Prozent der Interviews realisiert werden; 18,6 Prozent der Interviews wurden vollständig realisiert und waren zudem auswertbar. Dies entspricht einer Gesamtstichprobe von 27.762 Personen; davon entfallen 24.058 Interviews auf die Basisstichprobe und 3.704 auf die Aufstockungsstichprobe.

\subsection{Gewichtung und Hochrechnung der Ergebnisse unter Einbezug der Bildung}

Die Ergebnisse des vorliegenden Berichts beruhen auf den Daten des Deutschen Freiwilligensurveys und werden gewichtet dargestellt. Die Gewichtung zielt darauf ab, die Repräsentativität zu verbessern, um verallgemeinerbare Aussagen treffen zu können und die Grundgesamtheit möglichst gut abzubilden (Kiesl 2019). Verzerrende Faktoren der Stichprobenzusammensetzung (zum Beispiel eine disproportionale Ziehung nach Regionen) sowie ungleiche Teilnahmewahrscheinlichkeiten (zum Beispiel nach Alter) können durch Gewichtung ausgeglichen werden. Im Rahmen des Freiwilligensurveys wurden bisher die Gewichtungsmerkmale Bundesland, Gemeindegrößenklasse (BIK), Geschlecht und Altersgruppe herangezogen. Diese Gewichtung wird nun um das Gewichtungsmerkmal der schulischen Bildung ergänzt. Personen aus unterschiedlichen Bildungsgruppen nehmen mit unterschiedlicher Wahrscheinlichkeit an Umfragen teil; daher sind verschiedene Bildungsgruppen in den Daten über- beziehungsweise unterrepräsentiert. Insbesondere Personen mit höherer Bildung sind eher bereit, an Befragungen teilzunehmen als Personen mit niedrigerer Bildung (Engel \& Schmidt 2019). Da Menschen mit höherer Bildung anteilig häufiger freiwillig engagiert sind als Menschen mit niedriger Bildung, wurden durch die Nicht-Berücksichtigung der Bildung in der Datengewichtung in den bisherigen Berichten zum Freiwilligensurvey die Engagementquoten zwischen 1999 und 2014 überschätzt.

Durch den Einbezug der schulischen Bildung in die Gewichtung wird die Grundgesamtheit der Wohnbevölkerung Deutschlands ab 14 Jahren nun besser abgebildet. Die Gewichtung inklusive schulischer Bildung wird in diesem Bericht auf die Daten aller fünf Erhebungswellen von 1999 bis 2019 angewendet. Die Engagementquoten fallen durch dieses Vorgehen für alle Erhebungswellen niedriger aus als bisher berichtet, und zwar um jeweils drei bis vier Prozentpunkte (Tabelle 2-5; Simonson et al. 2017). Es ändert sich somit insgesamt das Niveau der Engagementquoten, der Trend 
Tabelle 2-5 Vergleich der Engagementquoten ohne und mit Berücksichtigung des Gewichtungsfaktors Bildung im Zeitvergleich 1999-2019

\begin{tabular}{lccccc}
\hline & FWS 1999 & FWS 2004 & FWS 2009 & FWS 2014 & FWS 2019 \\
\hline $\begin{array}{l}\text { Engagementquote ohne Berück- } \\
\text { sichtigung des Gewichtungsfak- } \\
\text { tors Bildung }\end{array}$ & 34,0 & 35,7 & 35,9 & 43,6 & 43,6 \\
$\begin{array}{l}\text { Engagementquote mit Berück- } \\
\text { sichtigung des Gewichtungsfak- } \\
\text { tors Bildung }\end{array}$ & 30,9 & 32,7 & 31,9 & 40,0 & 39,7 \\
\hline Differenz in Prozentpunkten & $-3,1$ & $-3,0$ & $-4,0$ & $-3,6$ & $-3,9$ \\
\hline
\end{tabular}

Quelle: FWS 1999, FWS 2004, FWS 2009, FWS 2014, FWS 2019, gewichtet, eigene Berechnungen (DZA).

bleibt jedoch im Wesentlichen bestehen. Die Gewichtung der Daten inklusive Bildung wirkt sich auch auf andere Ergebnisse aus.

Für Regionalanalysen wurde ein gesondertes Gewicht verwendet: Für die Analysen unterhalb der Bundeslandebene können nur Befragungsdaten von Freiwilligensurvey-Teilnehmenden verwendet werden, die explizit einer Zuspielung regionaler Informationen zu ihren Befragungsdaten zugestimmt haben. Da sich diese Zustimmungsbereitschaft nicht vollkommen zufällig auf alle im Freiwilligensurvey Befragten verteilt, wird diese leichte Verzerrung durch die Verwendung eines zusätzlichen Gewichts ausgeglichen, sodass gewichtete Regionalanalysen ebenfalls an die Randverteilung entsprechend dem Mikrozensus angeglichen sind.

Basierend auf den Gewichten wurden Hochrechnungsfaktoren errechnet, die den jeweils aktuellen Bevölkerungsstand des Befragungsjahres berücksichtigen und es ermöglichen, Aussagen in Form von absoluten Zahlen zu berichten. So lässt sich mithilfe von Hochrechnungsfaktoren beispielsweise ermitteln, wie viele Millionen Menschen ab 14 Jahren sich im Jahr 2019 freiwillig oder ehrenamtlich engagieren.

Auch bei der Regionalgewichtung und den Hochrechnungsfaktoren wird im aktuellen Bericht die Bildung berücksichtigt; auch hier kommt es also zu Abweichungen gegenüber früher berichteten Zahlen.

\subsection{Repräsentativität der Befragung}

Die Gesamtstichprobe des Freiwilligensurveys soll bei den Analysen Aussagen ermöglichen, die auf die Grundgesamtheit der Bevölkerung Deutschlands ab 14 Jahren verallgemeinerbar sind. Tabelle 2-6 gibt an, wie sich die Gesamtstichprobe des Freiwilligensurveys 2019 über grundlegende Merkmale der Befragten - Geschlecht, Alter, Bildung und Migrationshintergrund - verteilt. Die Verteilung dieser Merkmale im Freiwilligensurvey wird zum einen ungewichtet (Spalte ,FWS 2019 ungewichtet') und 
Tabelle 2-6 Verteilung zentraler Merkmale in der ungewichteten und der (bildungs-)gewichteten Stichprobe des Freiwilligensurveys 2019 sowie im Mikrozensus 2019, in Prozent

\begin{tabular}{|c|c|c|c|c|}
\hline & $\begin{array}{l}\text { FWS } 2019 \\
\text { ungewichtet }\end{array}$ & $\begin{array}{c}\text { FWS } 2019 \\
\text { gewichtet } \\
\text { ohne Bildung }\end{array}$ & $\begin{array}{c}\text { FWS } 2019 \\
\text { gewichtet } \\
\text { inklusive Bildung }\end{array}$ & $\begin{array}{c}\text { Mikrozensus } \\
2019\end{array}$ \\
\hline \multicolumn{5}{|l|}{ Geschlecht } \\
\hline Frauen & 52,5 & 50,9 & 50,9 & 50,8 \\
\hline Männer & 47,5 & 49,1 & 49,1 & 49,2 \\
\hline \multicolumn{5}{|l|}{ Alter } \\
\hline 14-29 Jahre & 10,1 & 20,1 & 20,1 & 19,4 \\
\hline 30-49 Jahre & 21,6 & 29,1 & 29,1 & 29,3 \\
\hline 50-64 Jahre & 34,3 & 25,8 & 25,6 & 26,7 \\
\hline 65 Jahre und älter & 34,1 & 25,0 & 25,2 & 24,6 \\
\hline \multicolumn{5}{|l|}{ Bildung* } \\
\hline noch Schüler:in & 2,0 & 3,4 & 3,7 & 3,5 \\
\hline niedrige Bildung & 16,2 & 17,4 & 30,5 & 34,0 \\
\hline mittlere Bildung & 32,3 & 29,2 & 28,1 & 28,8 \\
\hline hohe Bildung & 49,5 & 50,1 & 37,6 & 33,6 \\
\hline \multicolumn{5}{|l|}{ Migrationshintergrund ${ }^{* *}$} \\
\hline mit Migrationshintergrund & 16,8 & 22,7 & 25,2 & 23,8 \\
\hline ohne Migrationshintergrund & 83,2 & 77,3 & 74,8 & 76,2 \\
\hline
\end{tabular}

Quelle: FWS 2019; Mikrozensus 2019; eigene Darstellung (DZA).

* Bildungsabschlüsse werden in der amtlichen Statistik erst ab 15 Jahren erfasst, entsprechend sind für die Verteilung der Bildungsabschlüsse im FWS nur Personen ab 15 Jahren berichtet.

** Der Migrationshintergrund kann für den Mikrozensus aus technischen Gründen erst ab 15 Jahren ausgewiesen werden, entsprechend sind für die Verteilung des Migrationshintergrunds im FWS nur Personen ab 15 Jahren berichtet.

Hinweis: Abweichungen in den Prozentwerten ergeben sich durch das Auf- und Abrunden der Werte.

zum anderen gewichtet ohne Berücksichtigung der Bildung als Gewichtungsfaktor (Spalte ,FWS 2019 gewichtet ohne Bildung') sowie gewichtet mit Berücksichtigung der Bildung als Gewichtungsfaktor (Spalte ,FWS 2019 gewichtet inklusive Bildung') dargestellt. Die letzte Spalte (,Mikrozensus 2019') gibt mit den Daten der amtlichen Statistik die Verteilung für Geschlecht, Alters- und Bildungsgruppen sowie nach Migrationshintergrund in der Grundgesamtheit unter allen Privatpersonen in Deutschland $\mathrm{ab} 14$ Jahren wieder.

Beim Vergleich der ungewichteten Stichprobe mit der Verteilung im Mikrozensus 2019 hinsichtlich der Merkmale Geschlecht, Alter, Bildung und Migrationshinter- 
grund wird zum einen eine leichte Überbeteiligung der Frauen im Freiwilligensurvey deutlich. Zum anderen zeigt sich, dass die beiden jüngeren Altersgruppen der 14- bis 29-Jährigen und der 30- bis 49-Jährigen im Freiwilligensurvey 2019 anteilig deutlich seltener vertreten sind als in der Grundgesamtheit der Wohnbevölkerung Deutschlands ab 14 Jahren. Die beiden höheren Altersgruppen der 50- bis 64-Jährigen und der 65-Jährigen und Älteren dagegen haben im Freiwilligensurvey 2019 überproportional häufig an der Befragung teilgenommen. Auch Personen, die noch zur Schule gehen, sowie Personen mit niedriger Schulbildung haben seltener am Freiwilligensurvey 2019 teilgenommen, als es ihrem Anteil in der Grundgesamtheit entspricht, während Personen mit mittlerer und höherer Bildung im Verhältnis stärker vertreten sind.

Zum Ausgleich dieser Verzerrungen in der Zusammensetzung der Stichprobe sind Gewichtungsfaktoren berechnet worden (Details siehe in Abschnitt 2.8), die allen Befragten im Freiwilligensurvey 2019 einen bestimmten Wert zuweisen, der ihre Über- beziehungsweise Unterrepräsentation anhand zentraler Merkmale weitestgehend ausgleicht. Der Vergleich der gewichteten Stichprobe des Freiwilligensurveys ohne Berücksichtigung der Bildung mit der Verteilung im Mikrozensus 2019 zeigt, dass die Verteilung nach Geschlecht und Altersgruppen im Freiwilligensurvey sehr gut an die Randverteilung des Mikrozensus angepasst wird (Tabelle 2-6). In Bezug auf die Bildungsverteilung findet eine Annäherung der Verteilung im Freiwilligensurvey an die Verteilung im Mikrozensus erst nach Aufnahme der Bildungsinformation in die Gewichtung statt. Dennoch kann die Verzerrung der Stichprobe hinsichtlich der Zusammensetzung von Bildungsgruppen auch durch die Gewichtung inklusive Bildung nicht vollständig aufgehoben werden. Darüber hinaus zeigt sich: Obwohl in der Gewichtung der Faktor Migrationshintergrund nicht berücksichtigt wurde, da die hierfür notwendigen Informationen in den ersten drei Erhebungswellen nicht vergleichbar erhoben wurden, erfolgt durch die Gewichtung (sowohl ohne als auch inklusive Bildung) eine deutliche Annäherung der Verteilung nach Migrationshintergrund im Freiwilligensurvey an die Verteilung in der amtlichen Statistik.

\subsection{Analysestrategie und zentrale Differenzierungskriterien}

Bei den im Bericht dargestellten Ergebnissen handelt es sich um relative Häufigkeiten, dargestellt in Prozent (zum Beispiel die Engagementquote). Berichtet werden die relativen Häufigkeiten auf Basis der gültigen Fälle, die in der Regel ohne fehlende Werte wie ,weiß nicht' oder, verweigert' berechnet werden. Die Ergebnisse werden nicht nur für die Wohnbevölkerung Deutschlands ab 14 Jahren insgesamt, sondern auch für unterschiedliche Bevölkerungsgruppen (zum Beispiel nach Geschlecht oder nach Altersgruppen) dargestellt. Ist eine Bevölkerungsgruppe im Datensatz mit weniger als 50 Fällen vertreten (Prozentuierungsbasis $n<50$ ), wird der prozentuale Anteil nicht berichtet. 
Für den vorliegenden Bericht wurden alle Ergebnisse unter Einschluss der Bildungsinformation gewichtet. In den Analysen wurden Gruppenunterschiede (beispielsweise zwischen Altersgruppen) oder Unterschiede zwischen Erhebungswellen auf statistische Signifikanz getestet. Verwendet wurde ein Signifikanzniveau von $\mathrm{p}<$ 0,05. Ist ein Befund statistisch signifikant, so kann mit mindestens 95-prozentiger Wahrscheinlichkeit davon ausgegangen werden, dass ein festgestellter Gruppenunterschied auch in der Grundgesamtheit vorhanden ist. Ist ein Befund nicht statistisch signifikant, heißt das, dass höchstwahrscheinlich kein Unterschied zwischen den Bevölkerungsgruppen beziehungsweise den Erhebungswellen vorliegt und die Abweichungen zwischen den Prozentangaben zufällig entstehen. Die Signifikanztests erfolgten auf der Basis gewichteter Daten. Berichtet werden sowohl statistisch signifikante als auch nicht statistisch signifikante Ergebnisse.

Zentrale Differenzierungskriterien des Berichts sind Geschlecht, Altersgruppen, Bildung und Migrationshintergrund. Nachfolgend wird die Operationalisierung dieser vier Differenzierungskriterien beschrieben. Die Darstellungen weiterer im Bericht verwendeter Gruppierungsvariablen sind in den entsprechenden inhaltlichen Kapiteln zu finden.

Geschlecht: Im Freiwilligensurvey 2019 gab es erstmals die Möglichkeit, neben ,weiblich' und ,männlich' auch ,divers' als Geschlecht anzugeben, jedoch haben insgesamt nur drei Personen diese Kategorie angegeben. Die Anteile von Personen mit der Geschlechterangabe divers können somit aufgrund der geringen Fallzahl (Prozentuierungsbasis $\mathrm{n}<50$ ) nicht gesondert dargestellt werden, und auch bei der Datenweitergabe muss aus datenschutzrechtlichen Erwägungen auf eine gesonderte Ausweisung verzichtet werden. Für die Analysen und auch später für die Datenherausgabe werden Personen mit der Geschlechterangabe divers der Gruppe der Frauen zugerechnet. Auch für die Erstellung der Gewichtungsvariablen wurde die Kategorie divers der Kategorie weiblich zugeordnet, da es bisher für den diversen Geschlechtseintrag keine offiziellen Zahlen gibt, an die die Randverteilung angepasst werden könnte. Diese Fälle wurden der nach Fallzahlen stärksten Kategorie (es haben mehr Frauen als Männer an der Befragung teilgenommen) zugeordnet, um den Einfluss auf die Verteilung zu minimieren. ${ }^{3}$ Die geschlechtsspezifischen Befunde werden in diesem Bericht somit ausschließlich für zwei Gruppen - Frauen (inklusive Diverse) und Männer - dargestellt.

Ebenfalls muss für das Differenzierungsmerkmal Geschlecht die Erfassung im Freiwilligensurvey beachtet werden. Die befragten Personen bekommen keine Frage gestellt, welchem Geschlecht sie zugehörig sind, sondern die Eintragung wird zu Beginn des Telefonats durch die Interviewperson vorgenommen. Dabei besteht die

3 Eine Kennzeichnung der Geschlechterinformation als fehlend im Datensatz könnte ähnlich wie die Ausweisung als divers bei der vorliegenden geringen Fallzahl Rückschlüsse auf die Personen zulassen und ist daher aus Datenschutzgründen ebenfalls nicht umsetzbar. 
Möglichkeit, dass Interviewpersonen nachfragen, wenn sie sich bei der Einschätzung unsicher sind. Darüber hinaus ist eine Korrektur der Geschlechterangabe möglich, wenn sich eine befragte Person aufgrund der geschlechtsspezifischen Formulierungen, die in den Folgefragen verwendet werden, falsch angesprochen fühlt. Möglicherweise machen jedoch hiervon nicht alle Personen Gebrauch. Das Vorgehen kann somit dazu führen, dass Personen mit der Geschlechterangabe divers bereits während des Interviews der Kategorie männlich oder weiblich zugeordnet wurden, und sie in der Anzahl unterschätzt werden. Die Möglichkeit, das Geschlecht im Interview direkt zu erfragen, wurde nicht gewählt, da dieses Vorgehen von den Befragten in Telefoninterviews als merkwürdig empfunden wird und zu vermehrten Abbrüchen führen kann. ${ }^{4}$

Altersgruppen: In diesem Bericht werden vier Altersgruppen unterschieden: 14- bis 29-Jährige, 30- bis 49-Jährige, 50- bis 64-Jährige sowie 65-Jährige und Ältere. Der Vergleich zwischen den Altersgruppen macht es möglich, das freiwillige Engagement von Personen in unterschiedlichen Lebensphasen abzubilden.

Bildungsgruppen: Im Bericht wird zwischen vier Gruppen mit unterschiedlicher Schulbildung differenziert: Personen, die zum Befragungszeitpunkt noch eine allgemeinbildende Schule besuchen; Personen mit niedriger Schulbildung (Bildungsabschlüsse bis einschließlich Volks- und Hauptschule sowie ein Schulabschluss in der DDR nach der achten Klasse); Personen mit mittlerer Bildung (Schulabschluss entsprechend mittlerer Reife, das heißt Realschulabschluss, Abschluss nach der zehnten Klasse in der DDR oder Abschluss einer Pflichtschule im Ausland); und Personen mit hoher Schulbildung (Fachhochschulreife, Abitur, Abschluss der erweiterten Oberschule in der DDR oder einer weiterführenden Schule im Ausland). Um die Vergleichbarkeit mit früheren Erhebungswellen zu gewährleisten, wird auf eine Differenzierung anhand weiterführender Bildungsabschlüsse verzichtet, da diese in den ersten Erhebungswellen teilweise nicht, beziehungsweise nicht vergleichbar erhoben wurden.

Migrationshintergrund: In diesem Bericht wird das Engagement von Personen mit Migrationshintergrund im Vergleich zum Engagement von Personen ohne Migrationshintergrund betrachtet. „Eine Person hat einen Migrationshintergrund, wenn sie selbst oder mindestens ein Elternteil die deutsche Staatsangehörigkeit nicht durch Geburt besitzt" (Statistisches Bundesamt 2019: 4). Unterschieden wird darüber hinaus, entsprechend dem Konzept des Statistischen Bundesamtes (Statistisches Bundesamt 2019), zwischen vier Gruppen von Personen mit Migrationshintergrund: Menschen

4 Dieses Vorgehen wurde bereits im Pretest für die Erhebung des Freiwilligensurveys 2014 geprüft, aufgrund negativer Rückmeldungen der Interviewpersonen (siehe Schiel \& Quandt 2014) aber für die Hauptstudie wieder verworfen. 
mit eigener Migrationserfahrung ohne deutsche Staatsangehörigkeit; Menschen mit eigener Migrationserfahrung mit deutscher Staatsangehörigkeit; Menschen mit Migrationshintergrund, die in Deutschland geboren sind, aber keine deutsche Staatsangehörigkeit haben; und Menschen mit Migrationshintergrund, die in Deutschland geboren sind und eine deutsche Staatsangehörigkeit haben. Diese Kategorisierung kann auf Basis der Daten der Erhebungswellen 2014 und 2019 vorgenommen werden; in den vorherigen Wellen wurden die Informationen zum Migrationshintergrund nicht vergleichbar erhoben.

\subsection{Datennutzung}

Die Daten aller Wellen des Freiwilligensurveys können für wissenschaftliche Forschungszwecke in Form von Scientific Use Files (SUFs) nach Abschluss eines Datennutzungsvertrages kostenfrei über das Forschungsdatenzentrum des DZA (FDZDZA; www.fdz-dza.de) bezogen werden. Außerdem stellt das FDZ ausführliches Dokumentationsmaterial für alle Erhebungen zur Verfügung, wie beispielsweise die Interviewfragen in Form des Erhebungsinstruments (Kelle et al. 2021b), Kurzbeschreibungen für die SUFs, Codebücher und Variablenkorrespondenzlisten, und berät Datennutzende. In den Datensätzen des Freiwilligensurveys, die über das FDZ-DZA herausgegeben werden, sind standardmäßig die Gewichtungs- und Hochrechnungsvariablen sowohl mit als auch ohne Einschluss der Bildungsinformation enthalten. Wir empfehlen die Verwendung der Gewichtung einschließlich Bildungsinformation. Die Gewichtung ohne Bildungsinformationen sollte nur noch für Vergleiche mit bereits publizierten Freiwilligensurvey-Befunden verwendet werden.

\section{Literatur}

Deutscher Bundestag (2002). Bericht der Enquete-Kommission „Zukunft des Bürgerschaftlichen Engagements“. Bürgerschaftliches Engagement: auf dem Weg in eine zukunftsfähige Bürgergesellschaft (Drucksache 14/8900). Berlin: Deutscher Bundestag.

Engel, U., \& Schmidt, B.-O. (2019). Unit- und Item-Nonresponse. In: N. Baur \& J. Blasius (Hrsg.) Handbuch Methoden der empirischen Sozialforschung (S. 385-404). Wiesbaden: Springer VS.

Kelle, N., Burkhardt, L., Kausmann, C., Simonson, J., Schupp, J., \& Tesch-Römer, C. (2021). Auswirkungen von Referenzzeiträumen auf die Selbstangaben zum freiwilligen Engagement: Ergebnisse einer experimentellen Studie, SOEPpapers on Multidisciplinary Panel Data Research (Vol. 1125). Berlin: Deutsches Institut für Wirtschaftsforschung (DIW).

Kelle, N., Kausmann, C., Schauer, J., Lejeune, C., Wolf, T., Simonson, J., \& Tesch-Römer, C. (2021). Deutscher Freiwilligensurvey 2019. Erhebungsinstrument. Berlin: Deutsches Zentrum für Altersfragen. DOI: 10.5156/FWS.2019.D.oo1 
Kiesl, H. (2019). Gewichtung. In: N. Baur \& J. Blasius (Hrsg.) Handbuch Methoden der empirischen Sozialforschung (S. 405-412). Wiesbaden: Springer VS.

Schiel, S. \& Quandt, S. (2014). Deutscher Freiwilligensuvey 2014 (FWS). Pretestbericht. Bonn: infas Institut für angewandte Sozialwissenschaft.

Schiel, S., Ruiz Marcos, J., \& Fahrenholz, M. (2020). Methodenbericht. Deutscher Freiwilligensurvey 2019. Bonn: infas Institut für angewandte Sozialwissenschaft.

Simonson, J., Hameister, N., \& Vogel, C. (2017). Daten und Methoden des Deutschen Freiwilligensurveys. In: J. Simonson, C. Vogel \& C. Tesch-Römer (Hrsg.) Freiwilliges Engagement in Deutschland - Der Deutsche Freiwilligensurvey 2014 (S. 51-88). Wiesbaden: Springer VS.

Statistisches Bundesamt (2019). Bevölkerung mit Migrationshintergrund - Ergebnisse des Mikrozensus 2018. Wiesbaden: Statistisches Bundesamt.

Open Access Dieses Kapitel wird unter der Creative Commons Namensnennung Nicht kommerziell 4.0 International Lizenz (http://creativecommons.org/licenses/bync/4.0/deed.de) veröffentlicht, welche die nicht-kommerzielle Nutzung, Vervielfältigung, Bearbeitung, Verbreitung und Wiedergabe in jeglichem Medium und Format erlaubt, sofern Sie den/die ursprünglichen Autor(en) und die Quelle ordnungsgemäß nennen, einen Link zur Creative Commons Lizenz beifügen und angeben, ob Änderungen vorgenommen wurden.

Die in diesem Kapitel enthaltenen Bilder und sonstiges Drittmaterial unterliegen ebenfalls der genannten Creative Commons Lizenz, sofern sich aus der Abbildungslegende nichts anderes ergibt. Sofern das betreffende Material nicht unter der genannten Creative Commons Lizenz steht und die betreffende Handlung nicht nach gesetzlichen Vorschriften erlaubt ist, ist auch für die oben aufgeführten nicht-kommerziellen Weiterverwendungen des Materials die Einwilligung des jeweiligen Rechteinhabers einzuholen. 